\title{
Modelagem da Anomalia Magnética no Granito Silva Jardim
}

\author{
Antonio Nuno de Castro Santa Rosa ${ }^{1}$ \\ Nilton Correia da Silva ${ }^{2}$ \\ Natasha Gomes Santos Stanton ${ }^{3}$ \\ Renata Schimit ${ }^{3}$ \\ Universidade Federal do Rio de Janeiro/CT/NIDES 1, Geociências/UFRJ 3 \\ Universidade de Brasília/Campus Gama/Eng. Produção 2
}

\section{Resumo}

Este estudo representa a modelagem magnética do granito silva jardim utilizando anomalias Magnéticas geradas por prismas verticais. Os corpos modelados e seus respectivos campos são armazenados no banco de dado espacial, contendo seus atributos como: tipo geométrico de corpo, profundidade do topo, profundidade da base, largura, intensidade de magnetização, direção de magnetização, localização espacial, espaçamento entre corpos. Em seguida esses campos são normalizados em relação ao dado real obtido no campo, em seguida são fundidos utilizando as técnicas de fusão: Haar, Wavelets $\mathrm{D}(2,2)$, Pirâmide gradiente. Na fase de Classificação vamos utilizamos a Rede Neural Auto-organizável SOM (Self-Organized-Maps -SOM) que tem seus parâmetros otimizados pelo algoritmo Genético (Silva, 2001).

\section{Introdução}

Neste estudo determinamos diferentes campos magnéticos gerados pelas anomalias de fontes com diferentes parâmetros: tipo geométrico de corpo, profundidade do topo, profundidade da base, largura, intensidade de magnetização, direção de magnetização, localização espacial, espaçamento entre corpos [1],[4]. A estrutura de banco de dados espaciais permite com 0 modelo objeto relacional ter diferentes tipos de consultas e armazenar algumas dezenas de milhares de modelos.

$\mathrm{Na}$ fase seguinte os modelos são selecionados de forma aleatória para o conjunto de treinamento das Redes Neurais e os demais modelos são fundidos com dados reais de campo total. A escolha da técnica de fusão vai determinar o efeito de maior ou menos detalhe na imagem sintética do campo total.

$\mathrm{Na}$ última fase utilizamos os modelos fundidos com o conjunto de dados do treinamento nas Redes Neurais para realizar o mapeamento e a classificação das anomalias contidas nos respectivos campos.

\section{Modelagem}

Utilizamos o modelo do prisma vertical para o campo magnético com os parâmetros medidos no campo para o granito silva jardim.
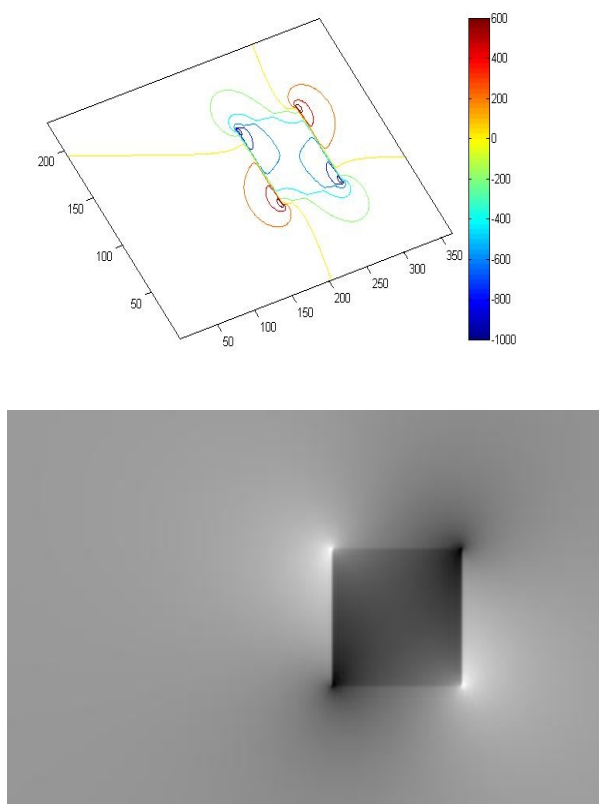

Figura 1. Modelo de prisma vertical para magnetometria com base infinita.

\section{Fusão}

A fusão da imagem observada com a gerada pelo campo magnético teórico foi realizada com três técnicas de fusão diferentes com segue:

Primeira utilizando a decomposição em Wavelets com base Haar entre as imagens do problema segue na figura 2 , baixo a imagem fundida.

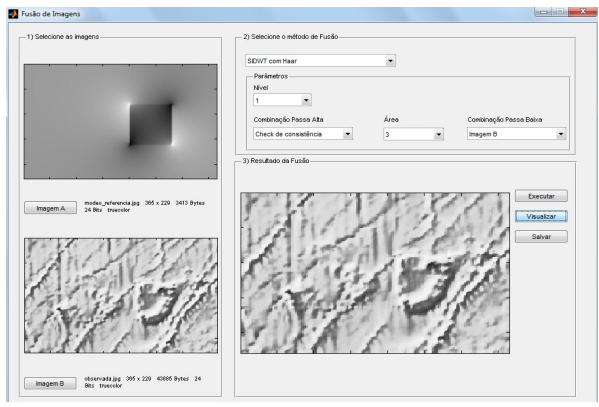


Figura 2. Fusão entre imagem do modelo e a imagem levantada em aerolevantamento a $150 \mathrm{~m}$ de altura. A segunda técnica de fusão utilizada foi a decomposição com Wavelets utilizando a base DWT com base DBSS $(2,2)$ como vista na figura 3 .

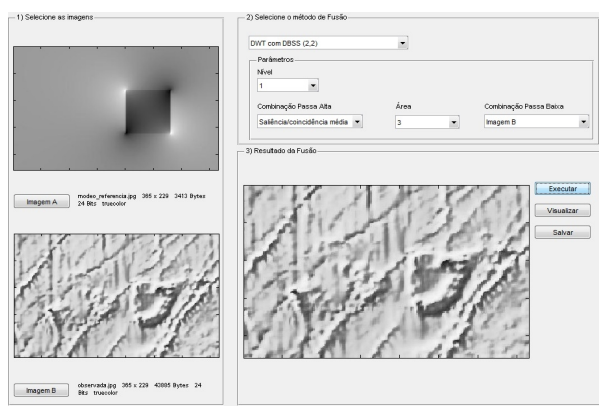

Figura 3. Fusão entre imagem do modelo e a imagem levantada em aerolevantamento a $150 \mathrm{~m}$ de altura.

$\mathrm{Na}$ terceira técnica de fusão utilizada foi a decomposição com o algoritmo de pirâmide gradiente como vista na figura 4.

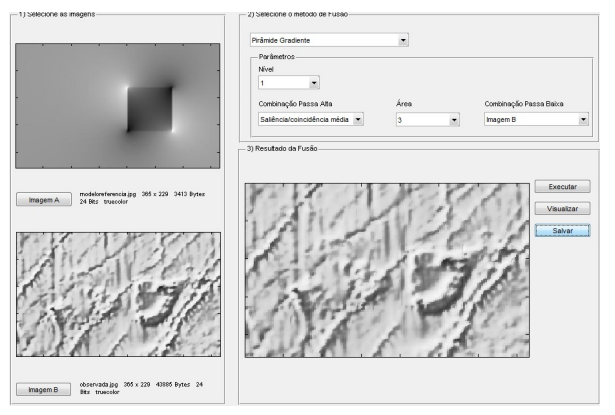

Figura 4. Fusão entre imagem do modelo e a imagem levantada em aerolevantamento a $150 \mathrm{~m}$ de altura.

Agora podemos trabalhar com as imagens geradas pelos processos das fusões em relação a imagem do aerolevantamento de magnetometria. A natureza desse problema é não linear devido o número grande combinações de parâmetros dos corpos que podemos obter para gerar um campo de forma a consegui o excelente ajuste em relação ao campo medido. Logo devemos utilizar a combinação do algoritmo não linear de Silva (2001) que apresenta o sistema GRN com algoritmo genético otimizando as redes neurais SOM [2]. Em seguida vamos apresentar os resultados conseguidos com esse sistema.

Em seguida vamos apresentar as parametrizações conseguidas com o sistema aplicado:
Primeiro para a imagem da fusão wavelet que utilizou a base Haar. Nas figuras 5 e 6, em seguida apresentamos a parametrização dos algoritmos de Síntese Genética para o caso da Rede Neural SOM.

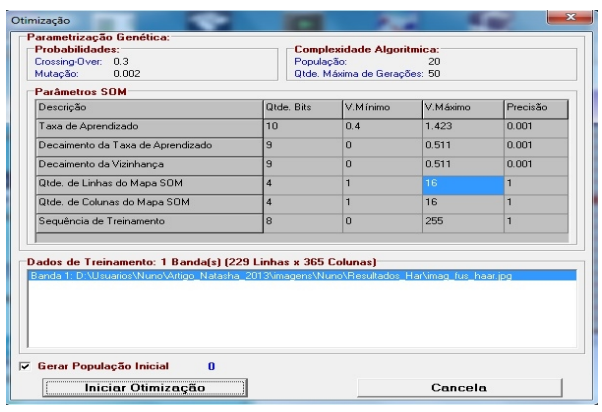

Figura 5. Parametrização do algoritmo genético com seu parâmetro de Crossing-Over iniciando em 0.3.

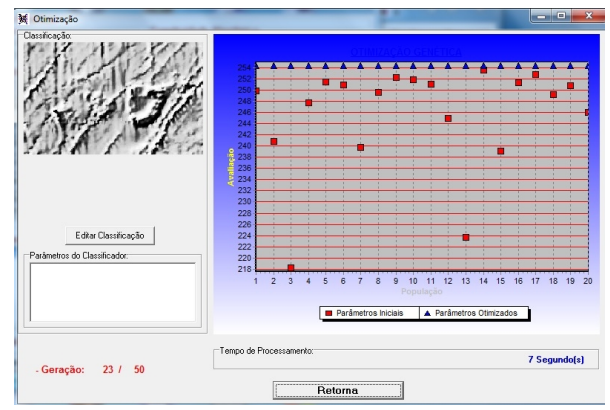

Figura 6. A imagem classificada utilizando a Rede Neural SOM [2], os parâmetros livre (retângulos vermelhos) que apresentaram um bom ajuste estão próximo dos valores de referencia (triângulos azul).

Em seguida apresentamos a parametrização para a imagem da fusão utilizando Wavelets utilizando a base DWT com base DBSS $(2,2)$ como vista na figura 3 . Veja nas figuras 7 e 8 os resultados,

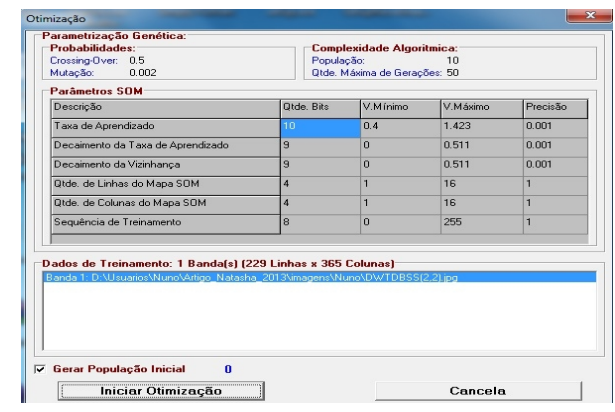

Figura 7. Parametrização do algoritmo genético com seu parâmetro de Crossing-Over iniciando em 0.4. 


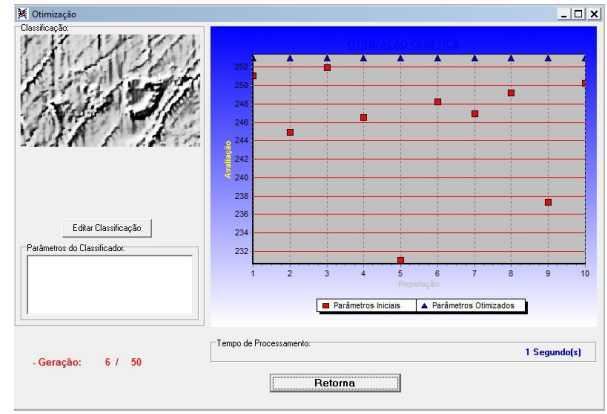

Figura 8. Os parâmetros livre (retângulos vermelhos) que apresentaram ajustes estão próximo dos valores de referencia (triângulos azul).

E por fim a parametrização para a imagem da fusão utilizando a decomposição com o algoritmo de pirâmide gradiente como vista na figura 4 . Os resultados estão nas figuras 9 e 10,

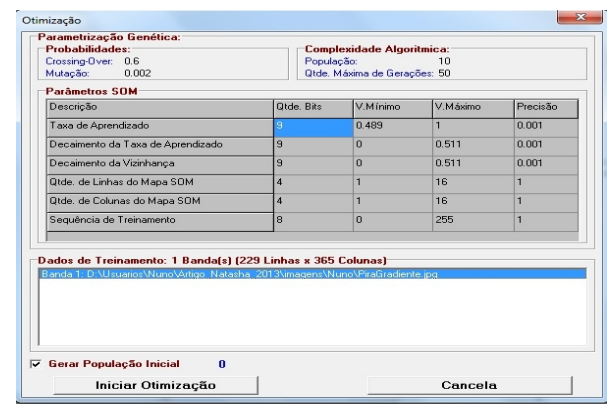

Figura 9. Parametrização do algoritmo genético com seu parâmetro de Crossing-Over iniciando em 0.48.

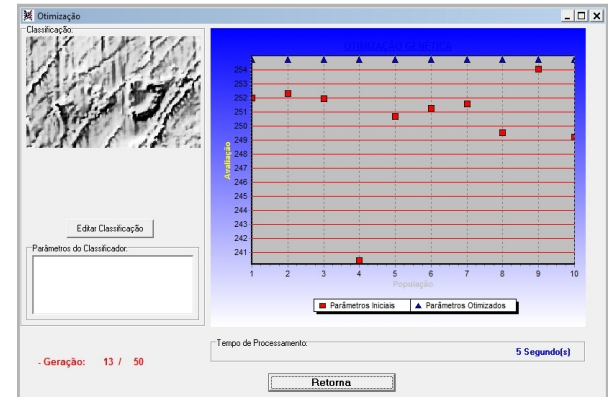

Figura 10. Os parâmetros livre (retângulos vermelhos) que apresentaram ajustes estão próximo dos valores de referencia (triângulos azul).

\section{Resultados}

As imagens que foram classificadas pelo algorítmo SGRN [3] [6] [7][8] [9] [10], gerou possíveis soluções que melhor se ajustam aos parâmetros do corpo estudado em seguida vamos visualizar as imagens para os respectivos métodos:

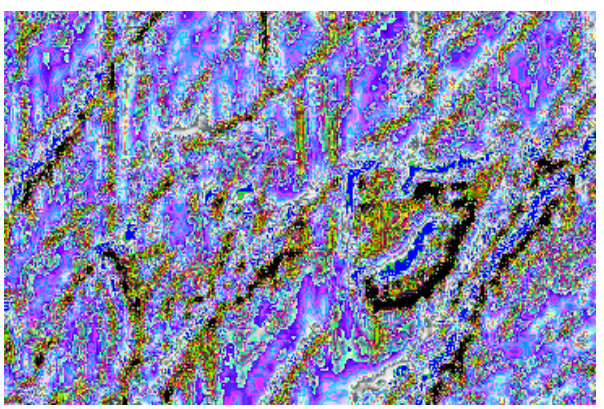

Figura 11. Imagem com origem com a decomposição wavelets e base Haar.

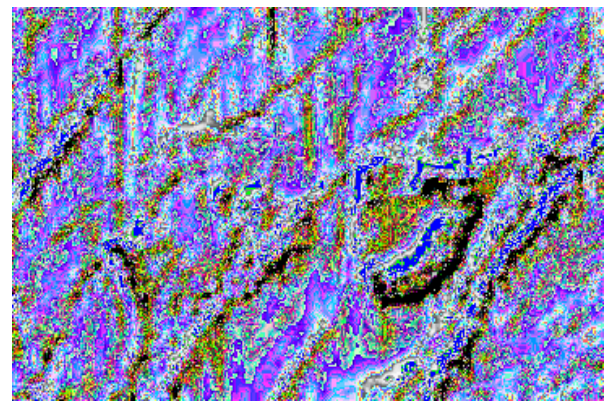

Figura 12. Imagem com origem com a decomposição wavelet e base DWT com base DBSS $(2,2)$.

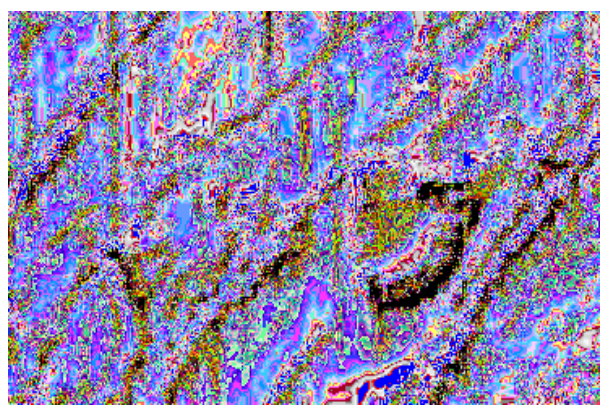


Figura 13. Imagem com origem com a decomposição com o algoritmo de pirâmide gradiente.

Em seguida realizamos o cálculo do número de bordas das imagens sintéticas em relação a imagem original para determinar qual desses resultados serve melhor para calculo de contatos. O método utilizado foi o que possibilita se ter qual dos resultados pode ser utilizado para calculo de área [5] [11].

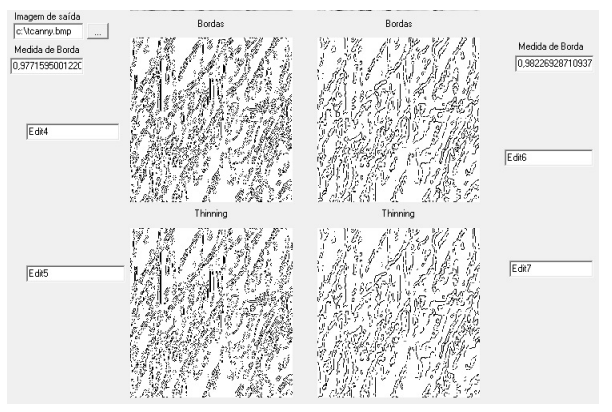

Figura 14. Imagem original comparada com imagem classificada com base Haar na figura 11.

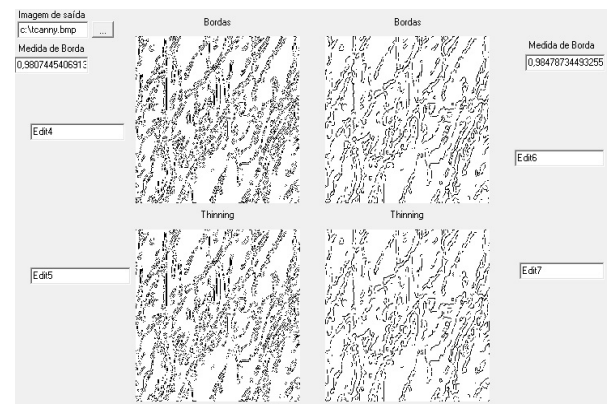

Figura 15. Imagem original comparada com imagem classificada com base DWT, DBSS $(2,2)$ na figura 12.

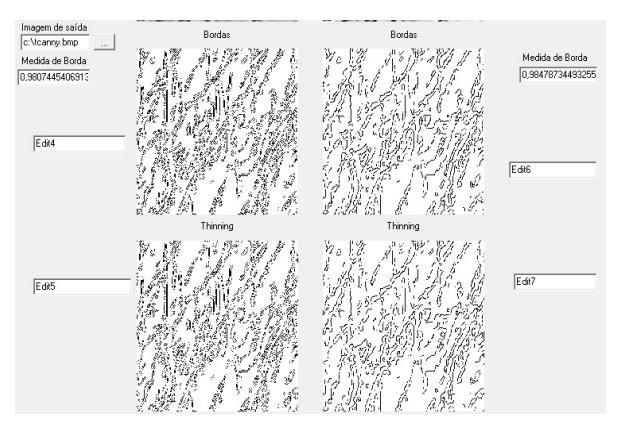

Figura 16. Imagem original comparada com imagem classificada com base Pirâmide gradiente figura 13.
Logo podemos com obter a imagem classificada que permite melhores medidas para determinação de contato em seu posicionamento das feições, para tal segmentamos a imagem classificada e foi separado as bordas que representam a anomalia magnéticas das outras falsas bordas. Afigura abaixo representa 0 resultado dessa segmentação.

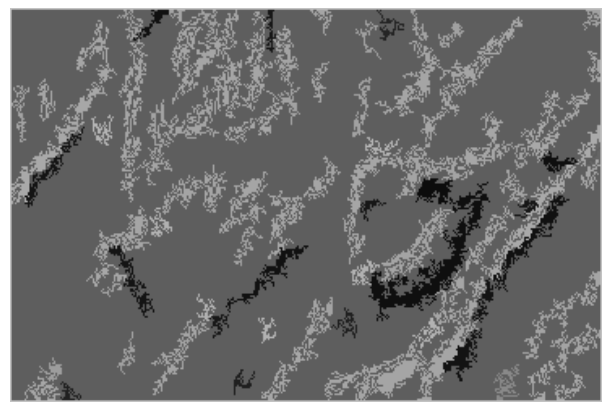

Figura 17. Imagem segmentada e que representa as melhores medidas a serem utilizadas para definição das bordas.

\section{Discussão e Conclusões}

Todos os resultados encontrados são sintéticos e com isto ainda possibilitam diferentes tipos de experimentos a serem realizados como: diferentes fontes, diferentes redes e dados observados de outras regiões.

A metodologia não depende da natureza da anomalia em si, porém do conjunto de treinamento utilizado na rede neural [3]. O Mapa Auto-Organizável, que é a rede possibilita diversas outras formas de analise ao problema abrindo assim grandes perspectivas de usa da mesma.

\section{Agradecimentos}

Agradecimento ao Instituto de Geociências da Universidade do Rio d Janeiro, laboratório de Petrofísica . Por fornecer os dados e o ambiente computacional. Os dados estão sob contrato e não podem ser divulgada a fonte.

\section{Referências}

[1] SILVA, N. C. ; ROSA, A. N. C. S. ; CARVALHO JUNIOR, O. A. ; Santos Junior, William Pereira dos . Classificadores Neurais Não Supervisionados. In: Genilda D'arc Bernardes; Roberto Prado de Morais. (Org.). Políticas Públicas: Meio Ambiente e Tecnologia. $1 \mathrm{ed}$. Goiânia: Vieira, 2010, v. 1, p. 207-217, ISBN: 9788589779722. 
[2] SANTA ROSA, A. N. C. ; WEIGANG, L. ; SILVA, N. C. ; MENESES, P.R. . Filtering and Classification of SAR images using Parallel Som. In: SPIE's 14th Annual International Symposium Aplications and Science of Computational Intelligence III, 2000, Orlando, Florida, U.S.A.. SPIE's 14th Annual International Symposium Aplications and Science of Computational Intelligence III. Orlando, Florida, U.S.A. : SPIE, 2000. v. 4055. p. 469475.

[3] SILVA, N. C. ; SANTA ROSA, A. N. C. . Estimative of SOM Learning Parameters Using Genetic Algoritms. In: 6th World Multiconference on Systemics, Cybernetics and Informatics (SCI 2002), 2002, Orlando, FL USA. 6th World Multiconference on Systemics, Cybernetics and Informatics (SCI 2002). Orlando , FL USA : SCI 2002, 2002. v. XX. p. 31-36.

[4] SANTA ROSA, A. N. C. ; SILVA, N. C. ; . Busca por modelo de prisma vertical do Campo Magnético utilizando rede neural: IV Simpósio de Geofísica Brasileira, Anais do simpósio, cidade Brasília,Brasil.

[5] SANTA ROSA, A. N. C. , Métodos de Fusão e Super Resolução de Imagens. Notas de Aula, Universidade do Rio de Janeiro, UFRJ/CT/NIDES, 2013, Rio de Janeiro, Brasil.

[6] Kohonen, T., Self-Organizating Maps, New York : Springer-Verlag, 1997.

[7] Kohonen, T., "Speech recognition based on topologypreserving neural maps ", (1990). "Neural Computings Architectures: The Design of Brain-Like Machines" Aleksander, I., pp. 26-40. MIT Press, Cambridge, Massachusetts, USA.

[8] Ritter H.; Schulten K.. "Convergence properties of Kohonen's topology conserving maps: Fluctuations, stability and dimension selection". Biol. Cybernetics, 60, pp. 59--71, 1988.

[9] Malsburg C. von der, "Network self-organization", In: An introduction to neural and electronic networks (S.F. Zornetzer, J.L. Davis, C. Lau, eds.), pp. 421-432. San Diego, CA: Academic Press, 1990.

[10] Haykin, S., "Neural Networks - A comprehensive foundation", Macmillan College Publishing Company, (1994), 352-354.

[11] Telles Júnior, Miguel Archanjo Bacellar Goes ; Santa Rosa, A. N. C. ; FONSECA, L. M. G. . Super-resolução de imagem QUICKBIRD-2 basada em algoritmo POCS e interpolação Sinc. Revista Brasileira de Cartografia (Impresso), v. 62, p. 1-4, 2010. 